\title{
Funciones cognitivas y desempeño académico en los estudiantes deportistas del gimnasio de la Universidad Técnica de Ambato
}

\section{Cognitive functions and academic performance in student athletes of the gymnasium of the Technical University of Ambato}

1 Jorge Rodrigo Andrade Albán https://orcid.org/0000-0002-7389-6624

Universidad Técnica de Ambato, Facultad de Ciencias Humanas y de la Educación, Carrera de Psicopedagogía. Ambato, Ecuador jr.andrade@uta.edu.ec

2 Noemí Rocío Andrade Albán Universidad Técnica de Ambato, Facultad de Ciencias Humanas y de la Educación, Carrera de Psicopedagogía. Ambato, Ecuador nr.jrandrade@uta.edu.ec

3 Jarrín Freddy Alcívar Jarrín https://orcid.org/0000-0002-7389-6624 Universidad Técnica de Ambato, Facultad de Ciencias Humanas y de la Educación, Carrera de Psicopedagogía. Ambato, Ecuador fa.jarrin@uta.edu.ec

4 Diana Carolina García Ramos $\quad$ (iD) https://orcid.org/0000-0002-7389-6624 Universidad Técnica de Ambato, Facultad de Ciencias Humanas y de la Educación, Carrera de Psicopedagogía. Ambato, Ecuador, dc.garcia@uta.edu.ec

Artículo de Investigación Científica y Tecnológica Enviado: $24 / 12 / 2021$

Revisado: $29 / 12 / 2021$

Aceptado: $12 / 01 / 2022$

Publicado:08/03/2023

DOI: https://doi.org/10.33262/concienciadigital.v6i1.4.2061

Andrade Albán, J. R., Andrade Albán, N. R., Alcívar Jarrín, J. F., \& García Ramos, D. C. (2023). Funciones cognitivas y desempeño académico en los estudiantes deportistas del gimnasio de la Universidad Técnica de Ambato. ConcienciaDigital, 6(1.4), 11701199. https://doi.org/10.33262/concienciadigital.v6i1.4.2061

CONCIENCIA DIGITAL, es una Revista Multidisciplinar, Trimestral, que se publicará en soporte electrónico tiene como misión contribuir a la formación de profesionales competentes con visión humanística y crítica que sean capaces de exponer sus resultados investigativos y científicos en la misma medida que se promueva mediante su intervención cambios positivos en la sociedad. https://concienciadigital.org

La revista es editada por la Editorial Ciencia Digital (Editorial de prestigio registrada en la Cámara Ecuatoriana de Libro con No de Afiliación 663) www.celibro.org.ec 
Palabras claves: funciones cognitivas, atención, memoria, funciones ejecutivas, desempeño académico, estudiantes universitarios

Keywords: cognitive functions, attention,

\section{Resumen}

En la educación superior siempre existen condiciones y factores que condicionan el desempeño académico y por ende obstaculizan el aprendizaje de los estudiantes, uno de ellos es el poco desarrollo de las funciones cognitivas. El funcionamiento cognitivo experimenta cambios importantes en estudiantes universitarios deportistas. La Universidad no debe limitarse sólo a la educación científica y técnica; sino debe ir más allá, buscar el desarrollo cognitivo, personal e integral de la persona del joven estudiante. El objetivo del presente estudio es analizar la relación entre funciones cognitivas y el desempeño académico de los estudiantes de la Universidad Técnica de Ambato. Metodología. Se aplica un enfoque mixto cuali cuantitativo. La modalidad de investigación es de campo y bibliográfica, porque la información fue recolectada de fuentes primarias. Además, tiene el carácter descriptivo, correlacional, de corte longitudinal, con un diseño no experimental y transversal. Resultados. Dentro de las funciones cognitivas afectadas en los estudiantes universitarios, la atención, la memoria de trabajo y funciones ejecutivas, constituye en un factor esencial que influye en distintos elementos cognitivos dentro de las habilidades académicas, hábitos de estudio y por ende en desempeño académico. En la evaluación de funciones cognitivas, se constata que el $75,68 \%$ de personas evaluadas poseen una atención y funciones ejecutivas "normal"; sin embargo, a nivel global se constata que un $23,42 \%$ presentan una alteración leve. Conclusión. En el análisis correlacional con el coeficiente Taub de Kendall se constata que existe una correlación media e inversa entre las funciones cognitivas y el desempeño académico, es decir mientras se constate más alteración en la memoria, atención y funciones ejecutivas, menor desempeño académico en los estudiantes universitarios. Es necesario la implementación y aplicación de métodos adecuados en las clases virtuales, para un mejor desempeño académico en los estudiantes.

\section{Abstract}

In higher education there are always conditions and factors that condition academic performance and therefore hinder student learning, one of them is the poor development of cognitive 
memory, executive functions, academic performance, university students functions. Cognitive functioning undergoes important changes in athletic university students. The University should not be limited only to scientific and technical education; If not, it must go further, seek the cognitive, personal, and integral development of the young student's person. The objective of this study is to analyze the relationship between cognitive functions and the academic performance of students at the Technical University of Ambato. Methodology. A qualitative and quantitative mixed approach is applied. The research modality is field and bibliographic because the information was collected from primary sources. It is also descriptive, correlational, longitudinal, with a non-experimental and cross-sectional design. Results. Among the cognitive functions affected in university students, attention, working memory and executive functions constitute an essential factor that influences different cognitive elements within academic skills, study habits and therefore academic performance. In the evaluation of cognitive functions, it is found that $75.68 \%$ of the people evaluated have "normal" attention and executive functions; however, globally, it is found that $23.42 \%$ present a slight alteration. Conclusion. In the correlational analysis with Kendall's Tau-b coefficient, it is found that there is a mean and inverse correlation between cognitive functions and academic performance, that is, while there is more alteration in memory, attention and executive functions, lower academic performance in the college students. It is necessary to implement and apply adequate methods in virtual classes, for better academic performance in students.

\section{Introducción}

La nueva sociedad del conocimiento del s. XXI y los cambios de la globalización, hace necesario que el sistema educativo universitario otorgue a la persona del universitario, las mejores herramientas que le permitan afrontar espacios de continuo crecimiento integral, de transformación e innovación; donde la formación se entienda como un proceso permanente para toda la vida y que requiere desarrollar un conjunto de capacidades y habilidades específicas, como por ejemplo, las funciones cognitivas. En esta línea, tiene gran importancia que los individuos puedan actuar de forma adecuada, efectiva y eficaz dentro de su marco referencial, con el fin de adaptarse al medio, desarrollar todas sus potencialidades y crecer integralmente como persona. Este proceso se logra mediante el 
fortalecimiento de distintas herramientas cognitivas, como la atención, memoria, funciones ejecutivas y la regulación de la conducta humana a partir de la motivación y los intereses personales.

Con la llega de la pandemia, el rol de cada integrante de la comunidad educativa universitaria sufre ciertas alteraciones psicoeducativas, siendo el estudiante el protagonista en el proceso educativo. No obstante, el cambio abrupto a una realidad de educación virtual deja entrever las precariedades en el diseño de políticas de estado para afrontar la era digital; debido a la crisis económica propia de la época. La gran mayoría de la población del país y América Latina experimenta dificultades para acceder a este tipo de escenarios de aprendizaje por la falta de dispositivos digitales, falta de conectividad, ausencia de herramientas de afrontamiento emocional frente al confinamiento, escaso conocimiento sobre el uso de las TICs, un limitado capital cultural en los padres de familia, entre otros.

En este contexto de la emergencia sanitaria provocado por el covid-19, todas las instituciones de Educación Superior (IES), están llamadas a promover el desarrollo de las funciones cognitivas en sus estudiantes, acompañado de un nuevo estilo de vida saludable de su población estudiantil; incorporar estrategias pertinentes que contribuyan a fortalecer el ámbito formativo del área intelectual, física y emocional; a través de la práctica deportiva. Bajo esta lógica, varios estudios señalan que es menester un seguimiento y monitoreo para conocer el real impacto de la actividad física en las funciones cognitivas básicas, las cuales se verán reflejados en el mejor rendimiento académico y los hábitos de vida del joven universitario.

\section{Funciones Cognitivas}

Los procesos cognitivos tienen vital importancia para el desarrollo de los procesos neuropsicológicos en la actividad humana del aprendizaje y el desempeño académico del estudiante. Incluyen una variedad de funciones mentales, tales como la atención, memoria, percepción, lenguaje y capacidad de solucionar problemas. Las funciones cognitivas describen el uso o manejo del conocimiento e integran los procesos que dan sentido a las señales sensoriales y a las acciones motoras, a medida que son codificadas neuronalmente. Además, al hablar de funciones cognitivas se hace referencia no solo a las mencionadas anteriormente, sino también al trabajo asociado que existe entre ellas, con la finalidad de llevar a cabo procesos cada vez más complejos en el individuo.

Según Tamayo et al. (2019) las funciones cognitivas no solo ayudan a encaminarse en las decisiones trascendentales que deberá tomar en su vida, tal como la elección de carrera u ocupación; sino también a manejar el tiempo libre; saber afrontar la presión de grupo de sus compañeros, con el fin de evitar involucrarse en las actividades de sustancias psicoactivas o psicotrópicas, manejar la impulsividad y el comportamiento agresivo. Por 
su parte, Mejía (2017) sostiene que las funciones ejecutivas se relacionan intrínsecamente con las funciones del lóbulo frontal, considerara como un conjunto de habilidades cognitivas que operan en el individuo para dar lugar a la consecución de un fin establecido con anticipación.

Por otra parte, Rico (2021) manifiesta que las funciones cognitivas además permiten en el individuo: el diseño de conducta, la autorregulación de los procesos para la consecución del objetivo a realizar, así como la flexibilidad y la organización de la tarea propuesta. Por lo tanto, se puede observar que las características más relevantes de las mismas son la regulación del self, la secuenciación, la flexibilidad, la respuesta de inhibición, la planificación, la memoria de trabajo y la organización de la conducta. Mientras que García \& González (2019) consideran que, en los últimos años, la pérdida de las funciones cognitivas forma parte de cuadros psicológicos, psiquiátricos y neurológicos como la migraña.

De Souza et al. (2019) prestan gran atención a la inteligencia, dejándose en evidencia que en la Neurología y ciencias anexas; se incluye la variable de inteligencia fluida y cristalizada, sin embargo, podemos anticipar que la inteligencia vista en la Psicología confluye aspectos cognitivos y funciones de áreas muy complejas en el cerebro humano.

Para Manriquez (2019) la memoria de trabajo y flexibilidad mental se asocian al rendimiento académico en edades inferiores, constituye un aporte significativo, porque permite continuar investigando el tema en un nivel educativo en el que no se ha realizado investigación al respecto, y del que el presente parece ser la primera experiencia en el área.

Por su parte Martínez et al. (2014) Las funciones ejecutivas engloban un amplio conjunto de funciones de autorregulación que permiten el control, organización y coordinación de otras funciones cognitivas, respuestas emocionales y comportamientos. El acercamiento tradicional a la hora de evaluar estas funciones, normalmente a través de las pruebas neuropsicológicos de lápiz y papel, puede mostrar en algunos pacientes un rendimiento mayor de lo esperado o dentro de los límites normales.

Acorde a lo mencionado anteriormente, se han realizado diversos estudios respecto a los trastornos cognitivos, que se presentan con mayor frecuencia después de una alteración del sistema nervioso central, son los problemas de atención y memoria presentes en niños y adultos y en población geriátrica (Ostrosky et al., 2012).

Atención. - La atención es un mecanismo cerebral que permite procesar los estímulos, pensamientos o acciones relevantes e ignorar los irrelevantes o distractores. Por tanto, Bernabéu (2017) considera que los mecanismos atencionales incluyen además una jerarquía de procesos, que van desde los niveles más generales e inespecíficos de 
activación cerebral, hasta los más concretos y diferenciados, como los implicados en tareas de atención alternante o dividida.

Atención Selectiva. - Es la habilidad para elegir los estímulos relevantes para una tarea, sin distraerse en estímulos irrelevantes (Belmar et al., 2013).

Atención Sostenida. - Habilidad para mantener la atención durante periodos prolongados. Capacidad necesaria para realizar tareas intelectuales, la misma que se puede alterar por causas orgánicas o emocionales (Rusca \& Cortez, 2020).

Control Atencional. - Incluye los procesos como la capacidad de planear y organizar la conducta y la inhibición de conductas inapropiadas (Reynoso \& Méndez, 2018).

Memoria. - Es un proceso que permite conservar la información transmitida por una señal después de que se ha suspendido la acción de esta, la memoria permite almacenar experiencias y percepciones para evocarlas posteriormente (Fierro et al., 2018).

Memoria Sensorial. - En este almacén llega a un órgano sensorial particular una gran cantidad de información que se retiene por breves instantes (décimas de segundo), ésta puede transferirse a la memoria a corto plazo o de lo contrario desaparecer rápidamente (Montero, 2015).

Memoria a Corto Plazo. - Se refiere a la evocación de la información inmediatamente después de su presentación (Valerio et al., 2016).

Memoria a Largo Plazo. - Se refiere a la evocación de la información después de un intervalo durante el cual la atención del paciente se ha enfocado en otras tareas (Flores \& Ostrosky, 2012).

\section{El desempeño académico}

La modalidad más reciente de formación educativa es la educación virtual, la cual implica la educación no presencial, inclinada al uso de tecnologías de información en el proceso de enseñanza y aprendizaje. La ventaja de este medio de enseñanza aprendizaje, es romper con la brecha de tiempo y espacio que es común en el entorno de educación presencial. No obstante, la pandemia por el Covid-19, obligó a las instituciones de educación superior a la aplicación de mecanismos digitales de manera apresurada y basado en consideraciones generales no acordes a la realidad de los estudiantes universitarios.

En varios estudios, en donde se realiza una valoración de los estudiantes a los entornos virtuales y la digitalización de esta en el contexto de la pandemia, es relativa, en relación con el alumno y al acceso a medio digitales de los mismos. La brecha en uso de medios tecnológicos depende del nivel de formación de la familia y el aspecto económico de la misma, que permite un seguimiento adecuado al proceso de enseñanza virtual. El limitado 
acceso a estas herramientas puede traer como consecuencia altos niveles de deserción académica en los jóvenes universitarios; teniendo en consideración que el educando no es un ser pasivo, sino un líder capaz de transformar su realidad desde sus experiencias y observación de modelos que influyen en sus motivaciones, emociones y valores personales.

En la actualidad las Instituciones de Educación Superior (IES), se han visto afectados en el proceso de enseñanza aprendizaje, por cuanto la modalidad presencial ha sido reemplazada por la educación virtual. Este nuevo contexto representa importantes cambios en la pedagogía y un reto en el uso de tecnologías digitales aplicadas a la educación superior. De manera que el contexto actual de la pandemia es una oportunidad para proponer el desarrollo de habilidades académicas en entornos sincrónicos y asincrónicos, los cuales han tenido efectos positivos en universidades de todo el mundo.

\section{Conceptualización de rendimiento académico}

El proceso de enseñanza y aprendizaje es uno de los más complejos que existen en la interacción humana y dentro de este se encuentra el rendimiento académico, con ello delimitarlo conceptualmente es difícil. Según Navarro (2003,) la complejidad del rendimiento o desempeño académico inicia desde su conceptualización, en ocasiones se le denomina aptitud escolar, desempeño académico o rendimiento escolar, pero por lo general las diferencias de concepto sólo se explican por cuestiones semánticas, sin embargo, también se le asocia con la medición cuantitativa de los conocimientos adquiridos y las capacidades desarrolladas por un estudiante durante un determinado periodo de tiempo. También supone la capacidad del estudiante para responder a los estímulos educativos.

Según Chilca (2017), el papel de docente es importante, asegura que debe identificar los principales problemas que afectan a los estudiantes en su proceso de aprendizaje, con la finalidad de obtener una buena o mala calificación; de manera que el rendimiento académico se lo mide o califica por medio de evaluaciones, las cuales cuantifican el conocimiento adquirido en clases por el educando.

\section{Factores del rendimiento académico en tiempos de pandemia}

Si bien es cierto existen diferentes conceptos relacionados al rendimiento académico; así como la presencia de varias propuestas para mejorar el mismo; existen varios factores que intervienen en el mismo y forman parte de las discusiones en diferentes ámbitos del sector educativo y social. Como afirma Chilca (2017), el proceso de educación es multidisciplinario, por lo tanto, la forma de evaluación también debería considerar varios factores en vista que ellos influyen directamente sobre el rendimiento académico, como el nivel intelectual, personalidad, motivación, aptitudes, interés, hábitos, autoestima, etc. 
Por otro lado, es menester indicar que en el nivel de educación superior amerita realizar un análisis desde múltiples perspectivas, en especial en tiempos de pandemia por el Covid-19 respecto al rendimiento académico. En este contexto influyen varios factores que definen el rendimiento académico, en vista que, en este nivel, no únicamente responde a las exigencias del docente sino también a las del grupo de estudio y las Tecnologías de la Información y la Comunicación (TIC).

Según Carranza (2017), mantener un buen rendimiento académico en la Universidad depende de asistir puntualmente a clases, cumplir con trabajos, participar en seminarios y trabajos grupales, además de la presión que tiene el educando en su hogar. Con ello el autor determina que en los primeros años de carrera el estudiante puede presentar estrés y cambios de ánimo, que afectan por ende el proceso de enseñanza aprendizaje. Por esta razón, se considera y está obligado el docente a reestructurar los espacios de aprendizaje a través de tecnologías virtuales.

Se conoce que otro de los factores que intervienen en el rendimiento académico, son los mecanismos que utilice el docente dentro del proceso de enseñanza y aprendizaje. Sin embargo, cabe indicar que el modelo de educación en donde el educando se sienta más involucrado en procesos multidisciplinarios a nivel de educación superior, en la que se desenvuelva de forma colaborativa, en convivencia con los demás; sigue siendo un desafío. Por lo tanto, es urgente la reestructuración de las aulas tradicionales en verdaderos entornos virtuales de aprendizaje, en donde se convergen todos los demás elementos o factores del desempeño académico como: los hábitos de estudio, la autoestima, el nivel intelectual, la personalidad, motivación, aptitudes, intereses, etc.

\section{Factores del bajo rendimiento académico}

Uno de los problemas que afronta el sistema educativo desde el nivel básico hasta el superior es el bajo rendimiento de algunos estudiantes y las pocas herramientas que disponen los docentes para gestionar el aprendizaje. Como asegura López (2015), el bajo rendimiento académico influye de manera especial en dos aspectos importantes cuando este afecta la autorrealización personal y profesional; y aquel que se ve limitado por no estar acorde a las exigencias del mundo profesional.

Datos recientes muestran que dentro del proceso del enseñanza y aprendizaje se han evidenciado algunos factores que influyen de manera directa en el rendimiento académico y la mayoría se relacionan con las herramientas metodológicas y la gestión de aprendizaje. Como asegura Valle (2018), en su estudio existen diversos factores que influyen en el rendimiento académico de los estudiantes en todos sus nivele, entre ellos tenemos:

- Nivel Socioeconómico

- Edad 
- Participación de los padres.

- Cantidad de deberes

- Género.

- Hábitos de estudio

- Acceso a medio tecnológicos.

- Curso

Este constructo del bajo rendimiento, según Carranza (2017), está relacionado con la autoestima de las personas, es de suponer que entre las principales causas del bajo rendimiento académico esta la falta de auto aceptación. Sostiene el autor, que en gran medida el rendimiento académico se relaciona también a la percepción que tienen las personas sobre su vida; por ello establece que dentro de los factores que condicionan y alteran el rendimiento académico, llama la atención el bienestar psicológico, entendido como el resultado de una evaluación respecto a cómo ha vivido.

\section{Causas del bajo rendimiento académico}

Una de las causas del bajo rendimiento académico tiene que ver con la autoestima de además de la madurez y educación emocional de cada persona, por lo cual las causas del bajo rendimiento académico son variadas. Como afirma Carranza (2017), estudios previos reportan que los estudiantes universitarios cuya edad va desde los 30 a los 55 años tienen un mayor rango de aceptación, dominio del entorno, crecimiento personal y propósitos en relación con los estudiantes jóvenes de entre 18 y 25 años. La auto aceptación juega un papel importante a la hora de desenvolverse en los espacios de debate y discusión de temas académicos estudiantiles.

Otra de las causas del bajo rendimiento académico está relacionada con los hábitos de estudios que desarrolla el estudiante dentro de su formación, en vista que la gestión del tiempo de estudio y la organización juega un rol fundamental. Según Valle (2018), en su estudio, refiere que la gestión del tiempo implica planificar y regular el uso del lapso tiempo destinado a la educación como refuerzo a lo revisado en el salón de clases. Si bien se dedica tiempo a la realización de tareas en la mayoría de los centros educativos, no se considera la calidad sino la cantidad de tarea. Finalmente, otros factores que generan un impacto negativo en el aprendizaje y por lógica en el rendimiento son: Los factores emocionales, condiciones ambientales, desmotivación, factores genéticos, etc.

\section{Consecuencias del bajo rendimiento académico}

Con la pandemia de Covid-19 se han presentado y profundizado falencias en las diferentes instituciones del Estado, incluso aquellas relacionadas al sistema de educación superior. Se identifica las contrariedades que presenta el sistema de educación en la colectivización 
del proceso de enseñanza aprendizaje, considerando que cada individuo aprende de manera diferente.

Con la finalidad de ayudar a los estudiantes que presentan problemas de rendimiento académico, es necesario conocer las principales características que presenta el estudiante en el proceso de enseñanza y aprendizaje. En casos extremos es necesario adaptar las técnicas de enseñanza a un nivel personalizado con el fin de evitar una baja en el rendimiento académico pese a ello se presentan algunos síntomas comunes del bajo rendimiento como son:

- Falta de asistencia escolares

- No realización de tareas

- Problemas emocionales constantes

- Falta de motivación generalizada.

\section{Tabla 1}

Consecuencias del bajo rendimiento académico

\begin{tabular}{ll}
\hline Perder el año escolar & $\begin{array}{l}\text { Las bajas calificaciones pueden ocasionar la pérdida del año } \\
\text { escolar por no cumplir metas }\end{array}$ \\
Analfabetismo & Los desertores no alcanzan destrezas lingüísticas \\
Discriminación & Los jóvenes enfrentan prejuicios de sus pares al no contar con \\
& recursos. \\
Incumplimiento de tareas & Los jóvenes desisten de continuar con sus metas y se vuelven \\
& sedentarios \\
Problemas emocionales & Son emocionalmente más susceptibles ante los retos. \\
Bajo nivel de persistencia & Los jóvenes pierden el interés por buscar soluciones o salir \\
& adelante. \\
Impulsividad & A menudo interrumpe a los demás y tiene dificultad para espera \\
Escasa atención & el turno
\end{tabular}

Fuente. Estudio realizado en la UTA, 2019. Elaboración: Equipo de investigación

\section{Entorno virtual y rendimiento académico}

El confinamiento por la pandemia de COVID-19 obligó a la humanidad a buscar formas de desarrollar sus actividades desde casa con la aplicación de herramientas virtuales tanto para el trabajo como para el estudio. Afirma Aguilar (2020) que "la telemática se convirtió en un nuevo canal de comunicación e información que dio lugar al teletrabajo y a la teleeducación". Como manifiesta la autora, esta forma nueva de actividades está presente y se considera que será implementada en algunos sectores tanto laboral como educativo.

Este sistema de aprendizaje por medio de la utilización de medio digitales no es nuevo, se ha venido utilizando con éxito dentro de modalidades a distancia y de manera especial en el sistema de educación privada. Con la pandemia esta modalidad ha crecido 
vertiginosamente no solo en el sector privado sino también en el entorno público y en todos los niveles.

Según Aguilar (2020), el contexto de la pandemia aceleró el paso en reemplazar los escenarios de aprendizaje presencial a virtual, para lo cual se requiere de las nuevas tecnologías de la comunicación y la información, herramientas muy importantes que tienen bondades tanto para el docente, como para el estudiante, como por ejemplo en los horarios flexibles, el acceso a los contenidos, comodidad de estudio entre otras cosas.

Además de la emergencia sanitaria por el Covid-19, la situación se complica más con el despido masivo de trabajadores del sistema educativo del sector público y privado; así como las desigualdades sociales que son evidentes entre aquellos que tienen posibilidades económicas y aquellos que a diario buscan medios para vivir. En este sentido se constata que el factor económico y la brecha digital se han convertido en los causantes de la deserción estudiantil de los últimos tiempos en la Universidad y en todos los niveles del sistema educativo nacional.

Cabe resaltar que, el uso correcto de los recursos tecnológicos en el proceso de enseñanzaaprendizaje universitaria pueden generar autonomía, criticidad y un aprendizaje participativo en el ser humano que aprende. El paso del aprendizaje en escenarios presenciales al aprendizaje virtual en tiempos de pandemia constituye un reto para la comunidad educativa, el rol del docente, los contenidos, el desarrollo de competencias y la evaluación deben partir desde la realidad del confinamiento social, para ello es preciso prestar atención al trabajo de las emociones para no caer en individualismos o en inútiles cosificaciones del ser humano.

Sin duda la llegada del internet ha marcado un hito en la utilización de medios tecnológicos en todos los entornos sociales; de manera especial en el campo de las telecomunicaciones aplicadas al entorno laboral y educativo. La oportunidad de acceder a información por medio herramientas digitales como la internet ha permitido un avance en el campo de la educación virtual y con ello la importancia de conocer las ventajas y desventajas que se presentan en esta nueva forma de desempeño social.

\section{Ventajas del entorno virtual de aprendizaje}

La educación en entornos virtuales no es nueva, se ha venido desarrollando en el sistema educativo, pero como soporte para el sistema presencial de educación, y como plataformas de intercambio de ideas e información. La pandemia ha fortalecido la utilización y desarrollo de las nuevas TIC's para la educación, que presenta herramientas beneficiosas. La educación virtual trae consigo beneficios a la comunidad educativa, permite desarrollar habilidades como la organización de información, el manejo de 
nuevos conceptos, la ampliación de lenguaje que favorece la comunicación y la conectividad (Aguilar, 2020).

Como se afirman varios docentes de educación a distancia, existen importantes ventajas de la educación virtual y la utilización de herramientas virtuales de aprendizaje entre ellas destacan: la flexibilidad de horarios, accesibilidad, autogestión del tiempo de estudio, calidad docente, ubicuidad, trabajo en equipo, evitar desplazamientos. Como se constata, existen diferentes formas de apreciar las ventajas que ofrecen las herramientas tecnológicas en el entorno educativo, es importante conocerlas y explotarlas en favor de nuestros intereses educativos.

Entre algunas ventajas tenemos: supera limitaciones de espacio y tiempo, amplia la cultura computacional, ahorro en viajes, amplía la cultura autodidacta, desaparece la masificación, el alumno puede seleccionar al profesor.

\section{Desventajas del entorno virtual de aprendizaje}

Si bien los entornos y las herramientas virtuales presentan un avance tecnológico en la educación, también es cierto que puede traer dificultades debido a la realidad social en especial de las clases vulnerables. Según asegura Aguilar (2020), ha existido una irrupción de forma violenta y sin previo aviso de estos medios tecnológicos en el entorno educativo sobre todo en los países que no se encontraban preparados para afrontar esta nueva realidad. Esto ha profundizado las brechas que existían en la región en el acceso a medios tecnológico.

Con relación a la experiencia educativa de los centros de educación superior, se constata que es variada en la utilización de herramientas tecnológicas; sin bien estas instituciones han aprovechado las ventajas que ofrece también han experimentado las desventajas que se presentan. Existen varias desventajas de la educación virtual por ejemplo soledad del alumno, exige constancia y disciplina. Como vemos las desventajas están más relacionadas a los hábitos de estudio de los estudiantes, con ello es importante reconocer las habilidades de cada uno para optar por la mejor opción, precio de implementación, se reduce el tiempo de las relaciones sociales, ritmo tecnológico es acelerado, motivación complicada.

\section{Retos del aprendizaje virtual y el rendimiento académico en tiempos de pandemia}

El proceso de aprendizaje y rendimiento académico en tiempos de pandemia por Covid19 es un reto para toda la comunidad educativa, por cuanto, el cambio repentino de escenarios presenciales de aprendizaje a un escenario virtual limita el contacto social del universitario, situación que trae consigo varias consecuencias, por ejemplo, la relación directa entre sujetos y dispositivos digitales, que evitan reconocer las emociones y sentimientos de los otros. De igual forma, las realidades educativas de los educandos 
difieren con cada sujeto, existiendo jóvenes universitarios con necesidades educativas especiales en los que el proceso de aprendizaje es incompleto, debido a la inadecuada adaptación curricular por parte de los docentes, quienes a su vez tienen dificultades para adaptarse a la realidad virtual.

Más allá de los desafíos que presenta la pandemia por Covid-19, es importante conocer las habilidades y destrezas que tiene cada estudiante para enfrentarse al entorno educativo. Tomar la decisión de estudiar con la modalidad virtual requiere una reflexión sobre las habilidades, destrezas, debilidades y hábitos. En ocasiones se puede optar por la modalidad mixta en la cual se conjuga la presencialidad con la utilización de mecanismos tecnológica, esta constituye una buena opción debido al acompañamiento presencial del docente.

El proceso de enseñanza aprendizaje tanto en su modalidad virtual como presencial presenta grandes retos en el manejo continuo de este proceso y la gestión del aprendizaje en tiempos de pandemia. Como afirma Aguilar (2020), en su estudio sobre la educación virtual que el tipo de aprendizaje generando por los espacios virtuales para el aprendizaje universitario, es aún precario si se considera que éste inicio en la última etapa del año lectivo, pero a pesar de ello, se pudo notar que los contenidos temáticos no pudieron ser debidamente abordados y profundizados en clases, no se hizo seguimiento del desarrollo de competencias de los estudiantes en todos los niveles de educación del país.

En definitiva, los retos de la educación virtual a nivel superior gira en torno a los siguientes aspectos: Conocer la realidad de los estudiantes, porque es imprescindible conocer qué requiere el estudiante y cómo se le puede hacer llegar el conocimiento que antes se impartía en el aula. De otro lado es importante aprovechar las herramientas digitales, pues, es tarea de las instituciones y del docente la actualización continua y el desarrollo de habilidades tecnológicas que faciliten el proceso educativo. Finalmente debe el docente y estudiante mantenerse comunicados; es decir con una comunicación ineficiente puede hacer que padres, docentes o personal directivo, no están alineados respecto al método educativo usado y lo necesario para que funcione el proceso académico.

\section{Herramientas digitales de enseñanza para un mejor rendimiento académico}

Las herramientas digitales son medios tecnológicos que se utilizan en varios aspectos como el laboral y educativo con el fin de suplir la necesidad de la presencia física de las personas en determinados lugares de trabajo o estudio. Como afirma Sánchez (2014), las herramientas tecnológicas "son todos aquellos software o programas intangibles que se encuentran en las computadoras o dispositivos, donde le damos uso y realizamos todo tipo de actividades". Como vemos es indispensable la utilización de herramientas digitales y acceso pleno a internet. 
La pandemia por COVID-19 ha fomentado la utilización de diferentes herramientas tecnológicas digitales en el campo educativo y laboral, lo que ha permitido que se desarrollen actividades virtuales de educación y teletrabajo. Además, el uso de estas herramientas digitales puede ser un problema para los docentes en especial aquellos que no acostumbran a utilizarlos. Esto produce también un retraso en el conocimiento de estas por parte de los estudiantes aumentado las brechas tecnológicas y digitales.

La utilización de estos medios digitales se ha diversificado para la utilización de estos en diversos aspectos como el laboral, educativo y comunicacional. Como afirma Sánchez (2014), las herramientas digitales tienen muy variados números y formas como:

- Medios de comunicación.

- Como forma de educación entretenida.

- Se permiten crear y llenar encuestas

- Permiten crear bases de datos

En tanto a las principales herramientas digitales de enseñanza que se utilizan en la educación virtual en tiempos de pandemia son: Zoom, Teams, Moodle, Issus, Evernote, Academia.edu

Finalmente, las estrategias educacionales utilizadas en todos los niveles permiten conocer los tipos de respuesta emocional de los estudiantes tanto en la educación básica, media y superior. Como afirma Valle (2019), actualmente las personas están expuestas a diversos tipos de información a cuál puede causar afectaciones las emociones y sentimientos de las personas. Esta información que es absorbida debe ser tratada por la conciencia de los estudiantes y es ahí donde interviene la inteligencia emocional y el manejo sentimental de los hechos.

\section{Metodología}

La metodología que se empleó en la investigación tiene un enfoque mixto, es decir cualitativo y cuantitativo, con un diseño no experimental, descriptivo y correlacional, que contribuyeron a la recolección de información y datos estadísticos. Para la investigación se seleccionó a 160 estudiantes deportistas de las diferentes carreras de la Universidad Técnica de Ambato. Para la variable independiente "funciones cognitivas ", se aplicó el Test Neuropsi y para la variable dependiente "Rendimiento académico" se aplicó un cuestionario que evalúa los factores que influyen en el desempeño académico. Para ello se conoció la relación existente entre las variables y se realizó un análisis e interpretación de los resultados obtenidos por medio de la aplicación de los instrumentos y se determinó que el 75,68\% de jóvenes cuentan con una atención y funciones ejecutivas "normal", lo cual significa los diferentes factores intrínsecos como afecciones de salud, motivación, estrés, entre otros y los factores extrínsecos de tipo familiar, social, cultural, etc. 
Los instrumentos utilizados para medir la variable independiente, las funciones cognitivas; se utilizó el Test Neuropisi, del autor original Ostrosky et al. (2012), es un instrumento de evaluación neuropsicológica breve que valora los procesos cognitivos de la memoria, atención y funciones ejecutivas, en este caso, se utilizó la subprueba con los ítems correspondientes para su retención y regresión. La forma de aplicación fue individual con un tiempo promedio de aplicación de una hora con veinte minutos.

Mientras que para la variable dependiente se utilizó el Cuestionario para evaluar factores del rendimiento académico, que es un cuestionario de elaboración propia, basado en el test de Artunduaga, con el fin de recopilar datos sobre los factores que influyen en esta problemática definida por las variables de estudio. Para el desarrollo del presenta manual, este se ha clasificado en varios factores como: Trayectoria en el Bachillerato, Trayectoria Universitaria, Variables Institucionales, Método de estudio, Percepciones sobre el rendimiento académico.

\section{Resultados}

A continuación, se presenta cada uno de los hallazgos obtenidos en la investigación, a través de la aplicación de los instrumentos: la prueba Neuropisi, del autor original Ostrosky et al. (2012), para evaluar factores del rendimiento académico basado en el test de Artunduaga.

\section{Tabla 2}

Población objeto de estudio

\begin{tabular}{ccc}
\hline $\begin{array}{c}\text { Total estudiantes } \\
\text { universitarios encuestados }\end{array}$ & Frecuencia & Porcentaje \\
\hline Hombres & 55 & $37 \%$ \\
Mujeres & 105 & $63 \%$ \\
Total & 160 & $100 \%$ \\
\hline
\end{tabular}

Fuente: Registro de estudiantes del gimnasio de la UTA.

Funciones cognitivas

Pregunta 1.- ¿Prefiere usted memorizar la materia antes de rendir una lección de forma oral o escrita en cualquier asignatura? 
Tabla 3

Memoria y rendimiento cognitivo

\begin{tabular}{ccc}
\hline Alternativas de respuesta & Frecuencia & Porcentaje \\
\hline Siempre & 17 & $15 \%$ \\
Casi siempre & 68 & $38 \%$ \\
A veces & 68 & $38 \%$ \\
Nunca & 7 & $9 \%$ \\
Total & 160 & $100 \%$ \\
\hline
\end{tabular}

Fuente: Cuestionario aplicado a estudiantes de la UTA

Figura 1

Nivel de memoria

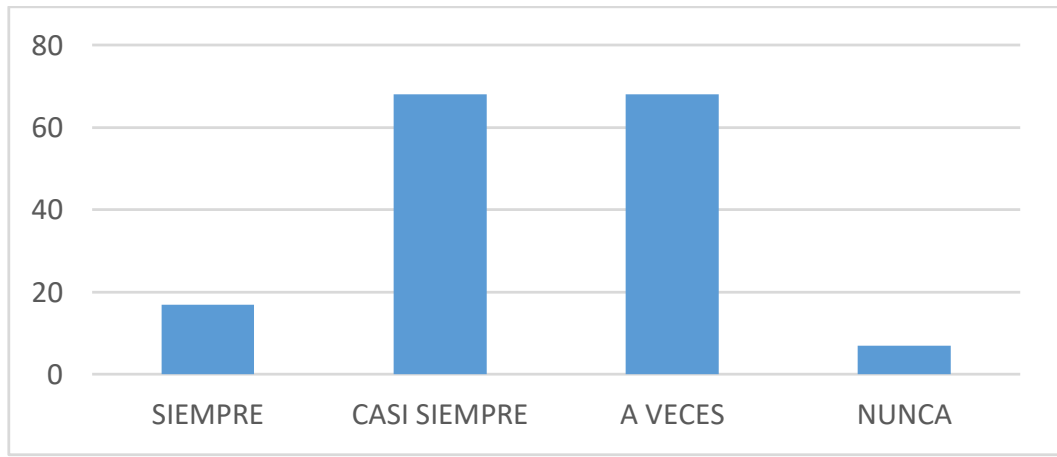

Fuente: Cuestionario aplicado a estudiantes de la UTA

De 160 personas evaluadas que corresponde al 100\% del estudiantado; reflejan que el $15 \%$ manifiestan que, siempre prefieren memorizar la materia antes de rendir una lección de forma oral o escrita en cualquier asignatura, el 38\% selecciona que casi siempre, el $38 \%$ manifiesta que a veces y el $9 \%$ de estudiantes mencionan que nunca. Se deduce que un alto porcentaje de estudiantes, prefieren casi siempre memorizar las materias antes de una lección oral o escrita, situación que no condiciona que obtengan calificaciones satisfactorias en relación con las personas que no tienen el hábito de memorizar los contenidos de la asignatura. De otro lado un grupo considerable, también señalan que a veces prefieren memorizar los contenidos de aprendizaje; pues, estos procesos cognitivos, les permite adquirir, retener y evocar diferentes tipos de conocimiento. Por tanto, este procedimiento le permite al universitario, organizar sus recursos cognitivos, afectivos y volitivos, al igual que organizar sus hábitos de estudio, el tiempo y espacio de desempeño académico. 
Pregunta 2.- ¿Muestra usted un alto nivel de concentración al momento de realizar una tarea o trabajo en el aula de clases?

\section{Tabla 4}

Nivel de concentración

\begin{tabular}{ccc}
\hline Alternativas de respuesta & Frecuencia & Porcentaje \\
\hline Siempre & 65 & $40 \%$ \\
Casi siempre & 73 & $46 \%$ \\
A veces & 21 & $13 \%$ \\
Nunca & 1 & $1 \%$ \\
Total & 160 & $100 \%$ \\
\hline
\end{tabular}

Fuente: Cuestionario aplicado a estudiantes deportistas de la UTA

\section{Figura 2}

Concentración

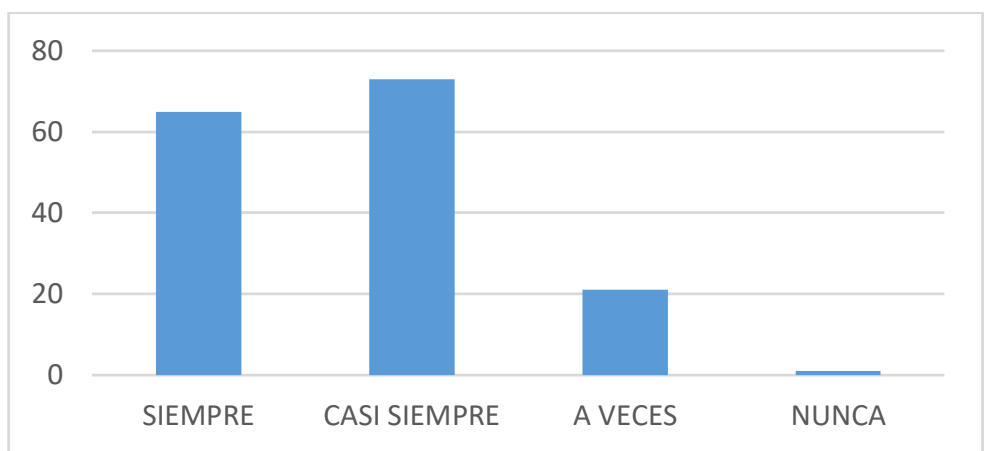

Fuente: Cuestionario aplicado a estudiantes deportistas de la UTA

De 160 personas evaluadas que corresponde al 100\% del estudiantado; reflejan que el $39 \%$, siempre muestra un alto nivel de concentración al momento de realizar una tarea o trabajo en el aula de clases; el $45 \%$ señala que casi siempre, mientras el $15 \%$ dice que a veces, y el $1 \%$ considera que nunca. Se establece que un alto porcentaje de estudiantes, prefieren casi siempre mostrar un alto nivel de concentración al momento de realizar una tarea en clases sobre alguna asignatura o sílabo. De otro lado un grupo considerable, también señalan que siempre demuestra concentración en los trabajos de aula.

Pregunta 3.- ¿Presta usted la atención adecuada a la explicación y a las indicaciones de sus actividades académicas dentro del aula de clases? 
Tabla 5

Nivel atencional

\begin{tabular}{ccc}
\hline Alternativas de respuesta & Frecuencia & Porcentaje \\
\hline Siempre & 60 & $37 \%$ \\
Casi siempre & 78 & $49 \%$ \\
A veces & 21 & $13 \%$ \\
Nunca & 1 & $1 \%$ \\
Total & 160 & $100 \%$ \\
\hline
\end{tabular}

Fuente: Cuestionario aplicado a estudiantes deportistas de la UTA

Figura 3

Nivel atencional

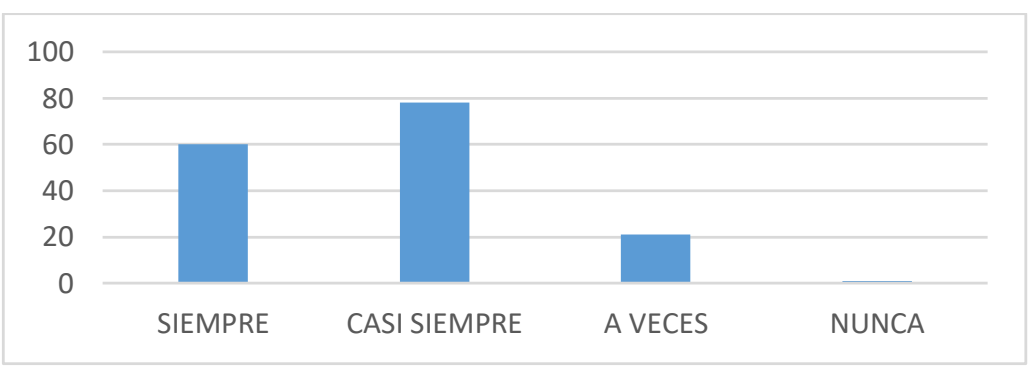

Fuente: Cuestionario aplicado a estudiantes deportistas de la UTA

De 160 personas evaluadas que corresponde al 100\% del estudiantado; reflejan que el 45\% siempre presta la atención adecuada a la explicación y a las indicaciones de sus actividades académicas dentro del aula de clases; el $46 \%$ señala que casi siempre presta atención a las indicaciones de sus docentes; mientras el $7 \%$ dice que a veces, y el 1\% considera que nunca. Se deduce que un alto porcentaje de estudiantes universitarios deportistas, siempre prestan la debida atención a la explicación de sus maestros y a las indicaciones que se les hace respecto a sus actividades académicas dentro del aula de clases y por ende las que debe desarrollarlas en el aula virtual sobre las diferentes unidades temáticas del sílabo en estudio. De otro lado un grupo considerable de esta población, también sostienes "casi siempre" prestan atención a la explicación que imprimen sus maestros y a las indicaciones sobre el desarrollo de actividades específicas del desarrollo académico en clases. De manera que los jóvenes en su mayoría están conscientes que esta función cognitiva es muy relevante para su proceso de aprendizaje académico, por lo que pese a tener muchos distractores en un espacio de aprendizaje presencial o virtual, saben cómo manejar los diferentes estímulos ambientales y focalizar su atención en las explicaciones e indicaciones que imparten sus maestros. 
El coeficiente de correlación con la primera pregunta es de - 0,651. Por lo tanto, hay una correlación media e inversa. Esto indica que mientras mayor sea la alteración en la memoria y atención los estudiantes menos prefieren memorizar la materia antes de rendir una lección.

A su vez, la correlación con la primera pregunta es de - 0,651. Por lo tanto, hay una correlación media e inversa. Esto indica que mientras mayor sea la alteración en la memoria y atención los estudiantes muestran un nivel más bajo de concentración al momento de realizar una tarea.

\section{Rendimiento Académico}

Trayectoria estudiantil durante el bachillerato

Tabla 6

Trayectoria estudiantil durante el bachillerato.

\begin{tabular}{lcccc}
\hline \multicolumn{2}{c}{ Si } & \multicolumn{2}{c}{ No } \\
\hline Pregunta & Frecuencia & Porcentaje & Frecuencia & Porcentaje \\
\hline P1 & 122 & $76,25 \%$ & 38 & $23,75 \%$ \\
P2 & 50 & $31,25 \%$ & 110 & $68,75 \%$ \\
P3 & 103 & $64,37 \%$ & 57 & $35,63 \%$ \\
\hline Fuen
\end{tabular}

Fuente: Encuesta a estudiantes de la Universidad Técnica de Ambato

\section{Figura 4}

Trayectoria estudiantil durante el bachillerato

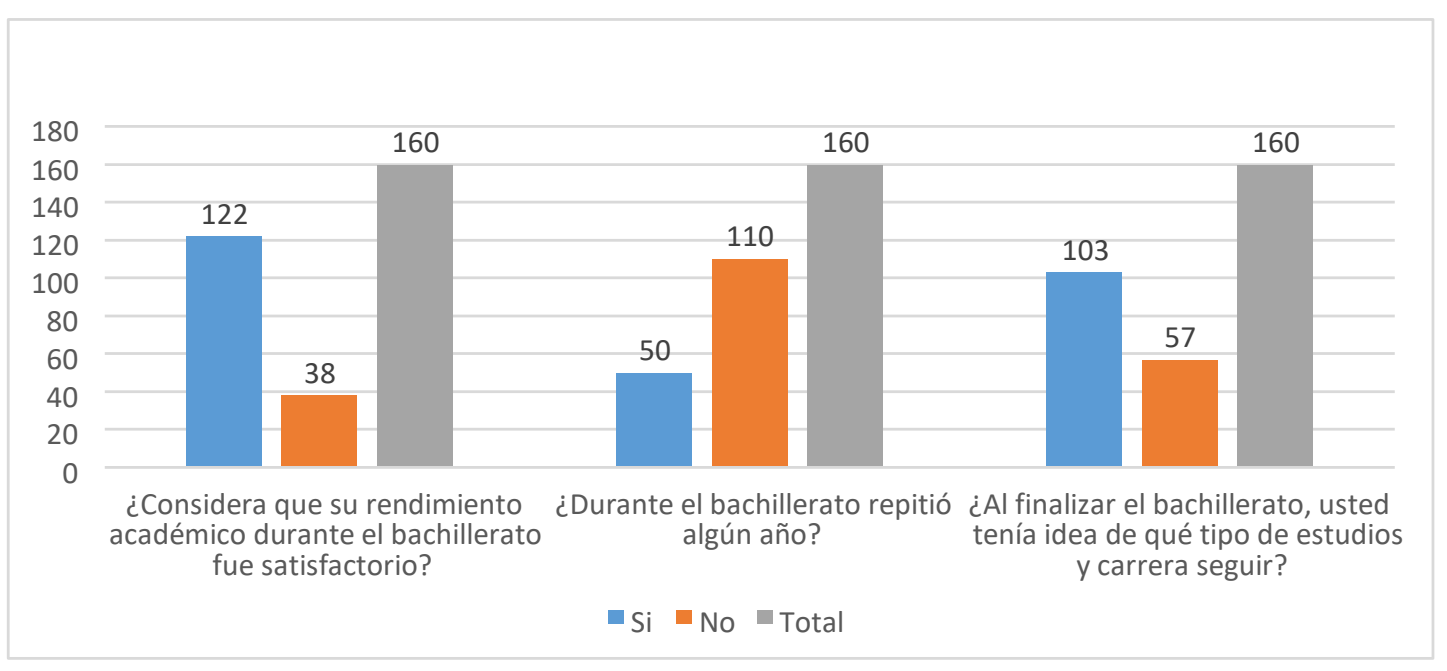

Fuente: Encuesta a estudiantes de la Universidad Técnica de Ambato 
Tras obtener los datos se determinó que en el componente Bachillerato, el 76,25\% de los estudiantes su rendimiento académico ha sido satisfactorio equivalente a 122 estudiantes mientras para el restante $23,75 \%$ equivalente a 38 estudiantes su rendimiento en el bachillerato fue deficiente. El $31.25 \%$ de los estudiantes de nivelación es decir 50 estudiantes han repetido un año durante el bachillerato; mientras que 110 estudiantes equivalente al $68,75 \%$ no ha repetido ningún año durante el bachillerato. En relación con la pregunta 3 se establece que para el 64,37\% equivalente a 113 estudiantes ya sabían qué carrera iban a estudiar, mientras que el $35,63 \%$ de los casos equivalente a 57 estudiantes no tenían conocimiento de la carrera que iban a estudiar en la universidad.

Trayectoria estudiantil universitaria

Tabla 7

Trayectoria estudiantil universitaria

\begin{tabular}{|c|c|c|c|c|}
\hline & \multicolumn{2}{|c|}{$\mathrm{Si}$} & \multicolumn{2}{|c|}{ No } \\
\hline Pregunta & \multicolumn{2}{|c|}{ Frecuencia Porcentaje } & \multicolumn{2}{|c|}{ Frecuencia Porcentaje } \\
\hline P4 & 62 & $38,75 \%$ & 98 & $61,25 \%$ \\
\hline P5 & 99 & $61,87 \%$ & 61 & $38,13 \%$ \\
\hline P6 & 111 & $69,37 \%$ & 49 & $30,63 \%$ \\
\hline
\end{tabular}

Fuente: Encuesta a estudiantes de la Universidad Técnica de Ambato

\section{Figura 5}

Trayectoria estudiantil universitaria

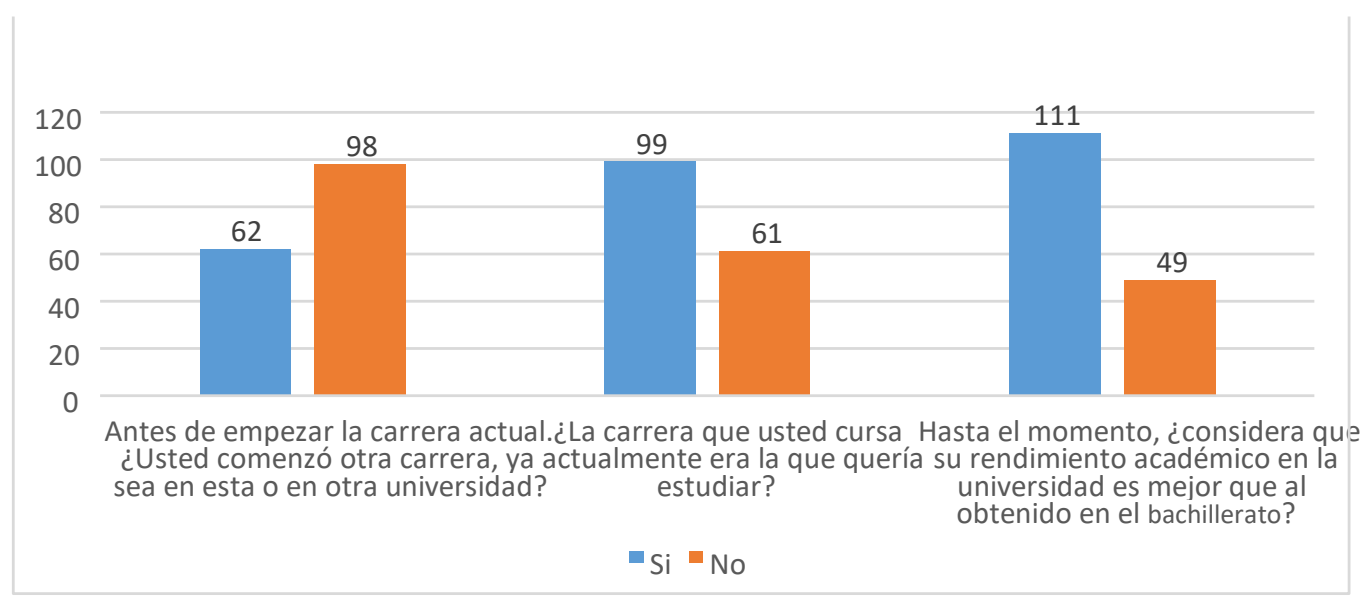

Fuente: Encuesta a estudiantes de la Universidad Técnica de Ambato 
Tras obtener los resultados en base al componente Trayectoria Estudiantil Universitaria, se determina que el 38,75\% equivalente a 62 estudiantes ya habían iniciado otra carrera universitaria de manera previa, mientras que el restante $61,25 \%$ equivalente a 98 estudiantes no han iniciado una carrera universitaria previamente. El 61,87\% equivalente a 99 estudiantes están en la carrera que querían estudiar, mientras que el 38.13\% restante equivalente a 61 estudiantes de los estudiantes encuestados se encuentran estudiando una carrera que no era su preferencia. El 69,37\% de los encuestados equivalentes a 111 estudiantes consideran que su rendimiento académico en la universidad ha mejorado en relación con el Bachillerato, mientras el 30,63\% equivalente a 49 estudiantes considera que su rendimiento no ha mejorado.

Variables institucionales y pedagógicas

\section{Tabla 8}

Variables institucionales y pedagógicas

\begin{tabular}{lcccc}
\hline & \multicolumn{3}{c}{ Si } & \multicolumn{2}{c}{ No } \\
\cline { 2 - 4 } Pregunta & Frecuencia & Porcentaje & Frecuencia & Porcentaje \\
\cline { 2 - 5 } P7 & & $77,50 \%$ & 36 & $22,50 \%$ \\
P8 & 124 & $84,38 \%$ & 25 & $15,62 \%$ \\
P9 & 135 & $76,25 \%$ & 38 & $23,75 \%$ \\
\hline \multicolumn{4}{r}{ Fuente: Encuesta a estudiantes de la Universidad Técnica de Ambato }
\end{tabular}

\section{Figura 6}

Variables instituciones y pedagógicas

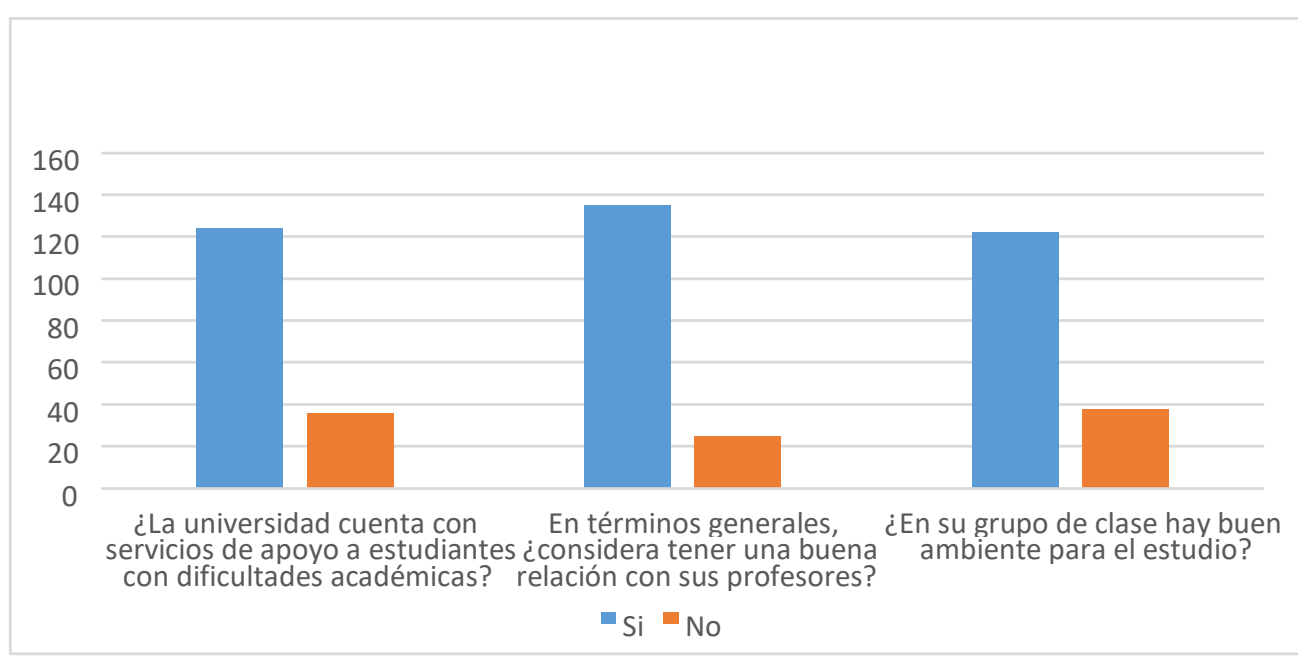

Fuente: Encuesta a estudiantes de la Universidad Técnica de Ambato 
Una vez se aplicado el instrumento con el fin de conocer los factores que influyen en el rendimiento académico se han recopilados los datos, los cuales poseen dos opciones de respuesta en este caso el componente Factores institucionales. Para el $77,50 \%$ equivalente a 124 estudiantes manifiestan que la universidad si cuenta con servicios de apoyo para estudiantes con dificultades académicas, mientas que el $22,50 \%$ restante equivalente a 36 estudiantes manifiestan que la universidad no presta los servicios necesarios para estudiantes en dificultades académicas. El $84,38 \%$ equivalente a 135 estudiantes establecen que existe una buena relación con los docentes, el 15,62\% equivalente a 25 estudiantes establecen que no existe una buena relación con los docentes. El 76.25\% de los encuestados equivalentes a 122 estudiantes establecen que en el grupo existe un buen ambiente de trabajo mientras que el $23.75 \%$ equivalente a 38 estudiantes establecen que el ambiente en el lugar de estudio no es adecuado.

Tabla 9

Método de Estudio

\begin{tabular}{lllll}
\hline & \multicolumn{2}{c}{ Si } & \multicolumn{2}{c}{ No } \\
\cline { 2 - 5 } Pregunta & Frecuencia & $\underline{\text { Porcentaje }}$ & $\underline{\text { Frecuencia }}$ & Porcentaje \\
\hline P10 & 97 & $60,63 \%$ & 63 & $39,37 \%$ \\
P11 & 87 & $54,37 \%$ & 73 & $45,63 \%$ \\
P12 & 138 & $86,25 \%$ & 22 & $13,75 \%$ \\
P13 & 73 & $45,62 \%$ & 87 & $54,38 \%$ \\
\hline
\end{tabular}

Fuente: Encuesta a estudiantes de la Universidad Técnica de Ambato

\section{Figura 7}

Variables Método de Estudio

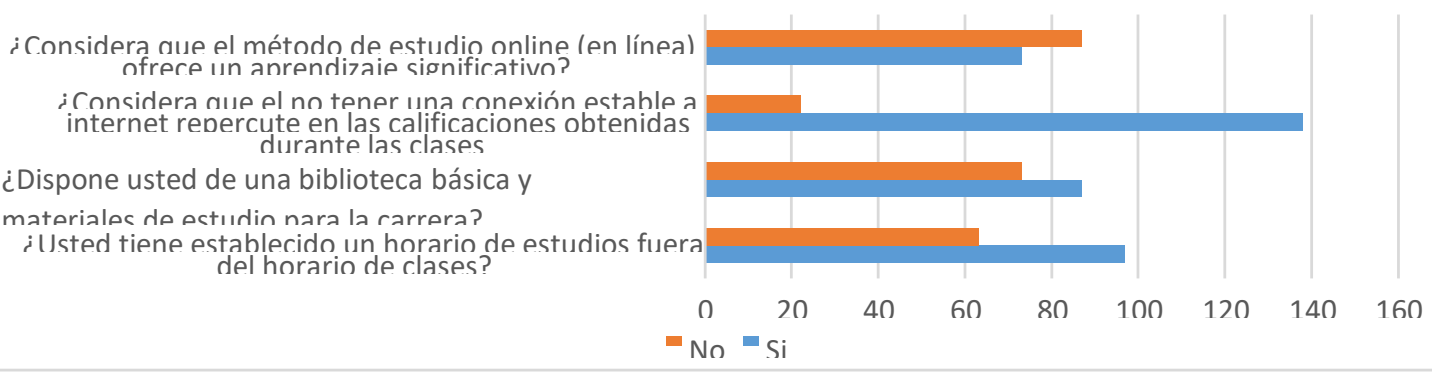

Fuente: Encuesta a estudiantes de la Universidad Técnica de Ambato 
De acuerdo con el componente métodos de estudio se determinó que el 60,63\% equivalente a 97 estudiantes no poseen un horario de estudios propio, mientras al 39,37\% equivalente a 63 estudiantes de los encuestados si posee un horario propio de estudio. El $54,37 \%$ equivalente a 87 estudiantes establecen que sí disponen de una biblioteca básica para poder estudiar con mayor facilidad mientras que el $45,63 \%$ equivalente a 73 estudiantes no disponen del mismo. Para el 86,25\% equivalente a 138 estudiantes consideran que al no tener una buena conexión a internet repercute directamente sobre las calificaciones de los estudiantes, mientras que el $13,75 \%$ equivalente a 22 estudiantes consideran que el acceso a internet no es un factor determinante en el rendimiento académico. El 45,62\% equivalente a 73 estudiantes consideran que el método de estudio online si ofrece un aprendizaje significativo, mientras que el 54,38\% equivalente a 87 estudiantes consideran que el método de estudios online no genera un aprendizaje significativo.

\section{Tabla 10}

Percepciones sobre el rendimiento académico.

\begin{tabular}{|c|c|c|c|c|}
\hline \multirow[b]{2}{*}{ Preguntas } & \multicolumn{2}{|c|}{ SI } & \multicolumn{2}{|c|}{$\mathrm{NO}$} \\
\hline & Frecuencia & Porcentaje & Frecuencia & Porcentaje \\
\hline P14 & 67 & $41,87 \%$ & 93 & $58,13 \%$ \\
\hline $\mathrm{P} 15$ & 122 & $76,25 \%$ & 38 & $23,75 \%$ \\
\hline
\end{tabular}

\section{Figura 8}

Percepciones sobre el rendimiento académico.

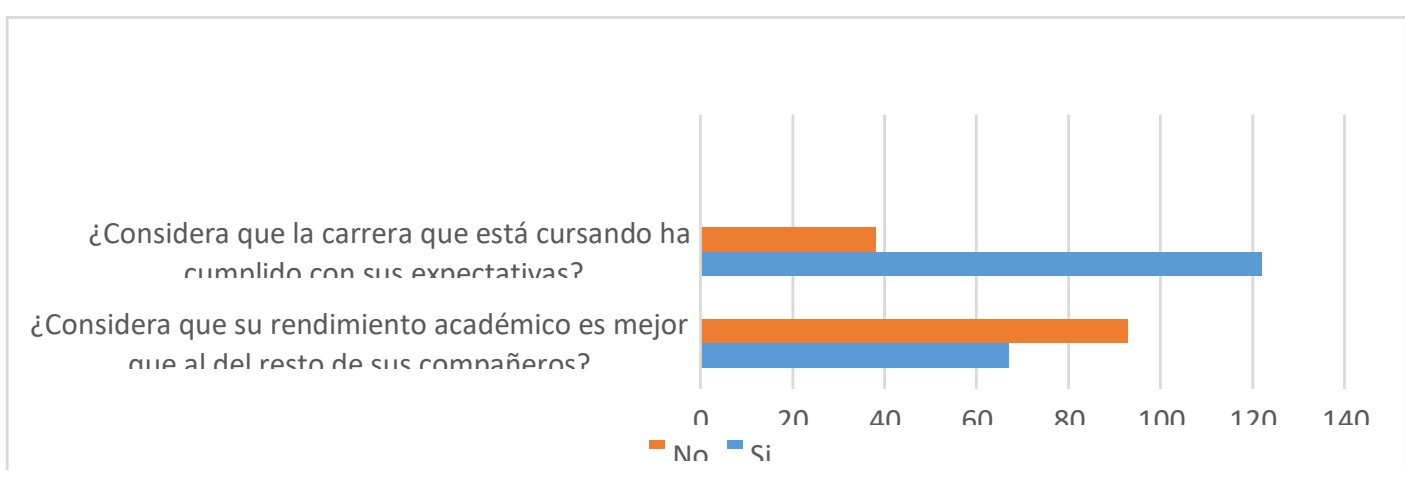

Fuente: Encuesta a estudiantes de la Universidad Técnica de Ambato 
De acuerdo con el componente Percepciones sobre el rendimiento académico se determina que el $41,87 \%$ equivalente a 67 estudiantes consideran que su rendimiento académico es superior que el de sus compañeros, mientras el 58,13\% equivalente a 93 estudiantes no consideran que su rendimiento sea superior al de sus compañeros. El $76,25 \%$ equivalente a 122 estudiantes consideran que la carrera ha superado sus expectativas mientras el $23,75 \%$ equivalente a 38 estudiantes consideran que la carrera no ha superado sus expectativas.

\section{Discusión}

En el país, los desafíos que enfrenta la educación superior son múltiples y variados; mismos que representan verdaderas oportunidades para buscar soluciones creativas desde una educación efectiva, humanística y comprometida, que potencialice las funciones cognitivas de la persona del universitario y por ende el desarrollo del conocimiento, porque el cambio de los países, sólo se podrá lograr desde la formación integral de sus estudiantes, de modo que posea un desempeño académico en el contexto del joven universitario en congruencia con las necesidades del contexto local y la práctica de su opción de formación profesional que tiene por delante.

En la investigación se encontró algunos aspectos que se asocia a las funciones cognitivas del estudiante universitario deportistas de la Universidad Técnica de Ambato, que gira en torno a la atención, memoria y funciones ejecutivas, en donde a nivel global se constata un 57, 66\% de estudiantes en los rangos normales, por lo que los mencionados procesos cognitivos, permiten un adecuado manejo del conocimiento y la adquisición de aprendizajes.

En lo que respecta a funciones ejecutivas Besserra et al. (2018), consideran que las FE son un factor clave para el éxito académico universitario y la implementación de instancias de apoyos pedagógicos favorecen el desarrollo profesional de los estudiantes. Por su parte Buterfuss \& Kendeou (2017), asocian la (FE) con el proceso de comprensión lectora, debido a que consideran que existen tres interacciones fundamentales entre: el lector, el texto y la situación del discurso.

Por lo tanto, no se concibe el aprendizaje con la ausencia o carencia de uno de los elementos antes mencionados. En contraste Cardona et al. (2018) comparten criterio al manifestar que los estudiantes, al momento de ingresar a la universidad, reconocen la importancia de la lectura en la formación personal y académica, pero pocos desarrollan un hábito lector y aplican estrategias de lectura adecuadas.

De otro lado en la población investigada de los estudiantes que acuden a practicar la actividad física en el gimnasio universitario, que cursan las diferentes carreras; se 
encontró a nivel global que solo un 23,42\% presentan una alteración leve en la atención, funciones ejecutivas y memoria.

En cuanto a la atención Li et al. (2015), conciben que esta función cognitiva se introduce en un marco de tres componentes que divide la atención en tres componentes distintos: alerta, orientación y control ejecutivo. La memoria se introduce bajo el modelo de tres componentes: memoria sensorial, memoria de trabajo o de corto plazo (MT) y memoria de largo plazo (MLP). Se pone énfasis en los componentes que muestran las interacciones más cercanas, como las interacciones entre la orientación y el control ejecutivo en el área de atención y entre MT y MLP.

De igual manera en esta investigación respecto a la atención y funciones ejecutivas, se encontró en la que solo el $23,42 \%$ de personas presentan una "alteración leve", lo que significa que la población que tiene dificultades en los procesos atencionales es aceptable. Teniendo en consideración esta función, es muy relevante para el desarrollo cognitivo, el control emocional y de aprendizaje del o la estudiante universitaria. Este resultado coincide con algunos estudios recientes asociados a la regulación de la conducta y las respuestas emocionales que promueven las funciones ejecutivas o las conductas dirigidas a metas, a veces referidas como regulación conductual o control cognitivo, que actualmente han sido de gran relevancia en el ámbito educativo. De esta manera se superaría algunos problemas asociados a las funciones ejecutivas, que implican una gran variedad de síntomas, como los errores perseverativos, la impulsividad, la falta de iniciativa, inflexibilidad mental, entre otros, como señala (Reynoso \& Méndez, 2018).

Desde un enfoque psicoeducativo, existe un creciente interés por el fenómeno de la metacognición en la educación superior, al aceptar que el desarrollar estrategias metacognitivas es una parte integral del proceso de aprendizaje del educando. Es que se deben potencializar para lograr el desarrollo de estrategias que conduzcan al estudiante a planificar, controlar y evaluar su propio progreso de aprendizaje ( $\mathrm{Al}$ et al., 2018).

Por último, es necesario señalar que las funciones cognitivas tienen una relación directamente proporcional con el mejorar el rendimiento académico del estudiante; toda vez que mejora los hallazgos de este estudio permiten establecer unas características cognitivas que evidencian deficiencias leves como en la comprensión de la información, la atención selectiva, memorización de temáticas, capacidad de planificación organizacional, etc. En definitiva, se constata un porcentaje considerable de funciones deficientes que tienen su impacto directo en otros procesos cognoscitivos, aunque no se ratifica completamente un perfil en particular en el estudiantado. De igual forma es necesario profundizar en los factores motivacionales y de aprendizaje, que permitirá ampliar el análisis respecto al rendimiento académico del universitario y precisar diferencias en el funcionamiento de los diversos dominios cognitivos desde un enfoque comparativo, explicativo y de tipo experimental con grupos de control. 


\section{Conclusiones}

- Los estudiantes universitarios que acuden a practicar la actividad física en el gimnasio de las diferentes carreras se encontraron a nivel global que un 23,42\% presentan una alteración leve en la atención, funciones ejecutivas y memoria. Se ha corroborado que las funciones ejecutivas, forman parte de las funciones cognitivas más complejas y que en los jóvenes cumple un papel fundamental en su aprendizaje, desarrollo cognitivo, toma de decisiones, actividades laborales, entre otras.

- En la evaluación de funciones cognitivas, se constata que el 75,68\% de personas cuentan con una atención y funciones ejecutivas "normal", lo cual significa los diferentes factores intrínsecos como afecciones de salud, motivación, estrés, entre otros y los factores extrínsecos de tipo familiar, social, cultural, etc., están siendo manejados de mejor adecuada, evitando que afecte a su bienestar y calidad de vida.

- En la atención y funciones ejecutivas, solo el 23,42\% de personas presentan una “alteración leve", lo que significa que la población que tiene dificultades en los procesos atencionales es aceptable. Teniendo en consideración esta función, es muy relevante para el desarrollo cognitivo, el control emocional y de aprendizaje del o la estudiante universitaria. Estos aspectos están asociados a la regulación de la conducta y las respuestas emocionales que promueven las funciones ejecutivas o las conductas dirigidas a metas de vida del individuo.

- Respecto a la función cognitiva de la memoria, en la investigación se encontró que en la frecuencia relativa del test Neuropsi, el 58,56\% oscila en un rango "normal", situación que le permite al estudiante universitario, conservar de forma actualizada una gran cantidad de información que debe procesar y recuperar en su diversidad de actividades académicas de aprendizaje y de su vida diaria.

- Los principales aspectos que influyen en el rendimiento académico son: la falta de orientación vocacional sobre la carrera a seguir, la relación existente entre docente-alumno, el no disponer de una biblioteca básica y materiales de estudio para la carrera, la deficiente conexión a internet del estudiantado, la falta de hábitos de estudio en cuanto al horario empleado fuera de las horas clases y finalmente consideran que la modalidad de estudios empleada actualmente (online) no ofrece un aprendizaje significativo. Es necesaria la implementación de técnicas y herramientas pedagógicas acorde al nivel de dificultad de los temas de clase; por ello es recomendable una capacitación docente en técnicas pedagógicas, entornos virtuales de aprendizaje y herramientas tecnológicas. 


\section{Referencias Bibliográficas}

Aguilar, F. (2020). Del aprendizaje en escenarios presenciales al aprendizaje virtual. Estudios Pedagógicos, 214.

Al, J. T., Mansor, N., Rashid, R., Bashir, I., \& Al-Jarrah, J. (2018). Actitud de los estudiantes de EFL hacia el uso de estrategias metacognitivas en la escritura. Canadian Center of Science and Education, 11(10), 162-171. Obtenido de https://files.eric.ed.gov/fulltext/EJ1192527.pdf

Belmar, M., Navas, L., \& Holgado, F. (2013). Procesos atencionales implicados en el trastorno por déficit atencional con hiperactividad (TDAH). Convergencia Educativa.

Bernabéu, E. (2017). La atención y la memoria como claves del proceso de aprendizaje. Aplicaciones para el entorno escolar. Reidocrea.

Besserra, D., Lepe, N., \& Ramos, C. (2018). Las Funciones Ejecutivas del Lóbulo Frontal y su Asociación con el Desempeño Académico de Estudiantes de Nivel Superior. Revista Ecuatoriana de Neurología.

Buterfuss, R., \& Kendeou, P. (2017). The Role of Executive Functions in Reading Comprehension. Educational Psychology Review.

Cardona, S., Osorio, A., Herrera, A., \& González, J. (2018). Actitudes, hábitos y estrategias de lectura de ingresantes a la educación superior. Educación y Educadores.

Carranza, R. (2017). Bienestar psicológico y rendimiento académico en estudiantes de. Revista de Investigacion en Ciencias Sociales, 135.

Chilca, M. (2017). Autoestima, hábitos de estudio y rendimiento académico en estudiantes universitarios. Propositos y representaciones, 4.

De Souza, J., Bezerra, C., Batista, E., Furtado, G., \& Isaza, C. (2019). Las células neurológicas y su interfaz en el proceso de aprendizaje de los seres vivos. Environmental Smoke.

Fierro, M., Aguinaga, L., Fierro, S., \& Ramos, C. (2018). Evaluación Neuropsicológica de funciones cognitivas en adolescentes indígenas escolarizadas. Talentos.

Flores, J., \& Ostrosky, F. (2012). Desarrollo neuropsicológico de los lóbulos frontales y funciones ejecutivas. México: Editorial El Manual Moderno.

García, E., \& González, X. (2019). Actualización en el manejo del vértigo. AMF. 
Li, S., Christ, J., Jhonson, N., \& Cowan, N. (2015). Attention and Memory. Brain Mapping.

López, P. (2015). Bajo rendimiento académico en estudiantes y disfuncionalidad familiar. MEDISAN.

Manriquez, L. (2019). Funciones ejecutivas y rendimiento académico en estudiantes de bachillerato dependiente de una casa hogar. Ride.

Martínez, G., Luna, P., Bombín, I., Cifuentes, A., Tirapu, J., \& Díaz, U. (2014). Evaluación neuropsicológica de las funciones ejecutivas mediante realidad virtual. Rev Neurol.

Mejía, G. (2017). Funciones Ejecutivas en niños y niñas de primaria: la importancia de las Inteligencias Múltiples como metodología de enseñanza-aprendizaje. $U A B$.

Montero, A. (2015). Efectos de los sentidos en la memoria sensorial . Scientia.

Navarro, R. (2003). El rendimiento académico: concepto, investigación y desarrollo. Revista Electrónica Iberoamericana sobre Calidad, Eficacia y Cambio en Educació, 4.

Ostrosky, F., Gómez, M. E., Matute, E., Rosselli, M., Ardila, A., \& Pineda, D. (2012). Neuropsi Atención y Memoria. México: Editorial El Manual Moderno.

Reynoso, O. O., \& Méndez, T. E. (2018). ¿Es posible predecir el rendimiento académico? La regulación de la conducta como un indicador del rendimiento académico en estudiantes de educación superior. Diálogos sobre educación. Temas actuales en investigación educativa.

Rico, P. (2021). Técnicas para un aprendizaje desarrollador en el escolar primario. Cuba.

Rusca, F., \& Cortez, C. (2020). Trastorno por déficit de atención con hiperactividad (TDAH) en niños y adolescentes. Una revisión clínica. Revista de Neuropsiquiatría.

Sánchez, E. (2014). Clasificación y funciones de las herramientas digitales. LEPRI, 2-3.

Tamayo, D., Hernández, J., \& Carrillo, S. (2019). Funciones ejecutivas en estudiantes de undécimo grado de colegios oficiales de Cúcuta y Envigado, Colombia. Universidad Simón Bolívar.

Valerio, G., Jaramillo, J., Caraza, R., \& Rodríguez, R. (2016). Principios de Neurociencia aplicados en la Educación Universitaria. Formación Universitaria. 


\section{DDigital}

\section{ISSN: 2600-5859}

Vol. 6 No. 1.4, pp. 1170 - 1199, marzo 2023

www.concienciadigital.org

Valle, A. (2018). Niveles de rendimiento académico e implicación en los deberes. European Journal of education and Psychology, 20.

Valle, M. (2019). El impacto de la inteligencia emocional en las aulas virtuales y su impacto socio educacional. ICONO, 505.

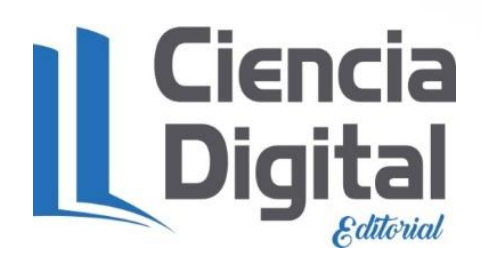


El artículo que se publica es de exclusiva responsabilidad de los autores y no necesariamente reflejan el pensamiento de la Revista Conciencia Digital.

\section{Ciencia \\ Ligital}

El artículo queda en propiedad de la revista y, por tanto, su publicación parcial y/o total en otro medio tiene que ser autorizado por el director de la Revista Conciencia Digital.
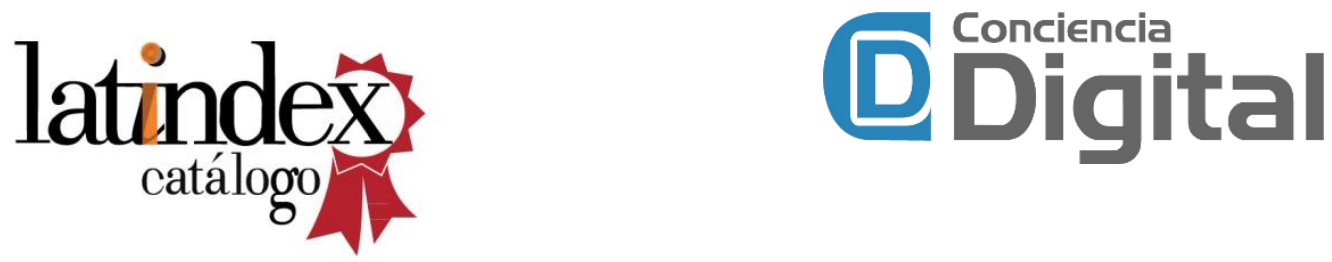

Indexaciones

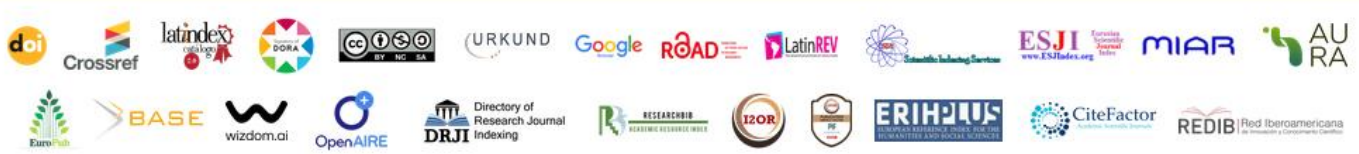

